\title{
SYNTHETIC POLYMERS WITH ANTICOAGULANT ACTIVITY
}

\section{VAN DER DOES, T. BEUGELING, P. E. FROEHLING, and A. BANTJES}

\author{
Department of Chemical Technology, Biomaterials Section, \\ Twente University of Technology, P. O. Box 217, Enschede, \\ The Netherlands
}

\section{SYNOPSIS}

Polymers with $\mathrm{C}=\mathrm{C}$ bonds have been modified by reaction with chlorosulfonyl isocyanate (CSI). Addition products were obtained in yields varying from $60 \%$ to $75 \%$. Reaction of these products with $\mathrm{NaOH}$ yielded polyelectrolytes with sulfamate and carboxylate groups, whereas from the reaction with $\mathrm{NH}_{4} \mathrm{OH}$ polyelectrolytes with sulfamate and carbonamide groups were obtained. The polyelectrolytes showed anticoagulant activity depending on the structure and on the presence of both sulfamate and carboxylate groups. These groups are essential for the anticoagulant activity, because $\mathbf{N}-\mathrm{S}$ bond cleavage in the sulfamate groups as well as substitution of carboxylate by carbonamide groups resulted in loss of activity. From the recalcification times of bovine plasma, it could be concluded that the most active polyelectrolyte had an activity of $6-7 \%$ compared with heparin. However, determination of the activated clotting time of blood from rabbits showed that this compound had an in vivo activity of $50 \%$ in comparison with heparin.

\section{INTRODUCTION}

The formation of $N$-chlorosulfonyl $\beta$-lactam groups in polymers by the addition of chlorosulfonyl isocyanate (CSI) to $\mathrm{C}=\mathrm{C}$ bonds has been reported [1-3]. It was shown that opening of the four-membered $\beta$-lactam rings by reaction with nucleophiles resulted in the formation of water-soluble polyelectrolytes with anticoagulant activity $[4,5]$. It was also found that polymer surfaces coated with one of these polyelectrolytes, when exposed to blood, showed reduced platelet adhesion [6].

In this paper modifications of several other polymers will be reported and their anticoagulant activities will be discussed in relation to their structure.

The starting polymers were poly(cis-1,4-isoprene) (I), poly(trans-1,4-isoprene) (II), poly(3,4-isoprene) (III), and styrene-isoprene-styrene block copolymers, $14 \%$ and $29 \%$ styrene by weight [(IV and (V), respectively].

These polymers were modified by reaction with CSI (Scheme 1) and the addition products were subsequently converted with $\mathrm{NaOH}$. Furthermore the reaction of some of the polymer-CSI adducts with $\mathrm{NH}_{4} \mathrm{OH}$ has also been studied. 


\section{EXPERIMENTAL}

For the reaction with CSI the polymers were dissolved in toluene (I, II), benzene (III), or cyclohexane (IV, V) $(0.10$ basal mole in $500 \mathrm{ml})$. The polymer solutions were added to a stirred solution of CSI in the same solvent $(0.15$ mole in $100 \mathrm{ml})$. The addition reaction was carried out at room temperature in a nitrogen atmosphere. After 15-30 min the solutions became turbid and precipitates were formed. Stirring was continued for $1 \mathrm{hr}$ and then the polymer-CSI adducts were isolated and washed with diethylether (Ia, IIa, IIIa, IVa, and Va).

The reaction of the polymer-CSI adducts with $\mathrm{NaOH}$ was carried out by heating these adducts with an excess of aqueous $\mathrm{NaOH}(2-2.5 M)$. Because of foaming, the mixtures were cautiously heated to boiling, and after $1 \mathrm{hr}$ clear, viscous solutions resulted. The reaction products could be isolated by precipitation in a five- to tenfold excess of ethanol (Ib, IIb, IIIb). After reaction of $\mathrm{IVa}$ and $\mathrm{Va}$ with $\mathrm{NaOH}$ no precipitate could be obtained upon addition to ethanol. It was possible, however, to isolate IVb and $\mathrm{Vb}$ by precipitation with concentrated $\mathrm{HCl}$.

The conversion of some of the polymer-CSI adducts (Ia and IIa) with $\mathrm{NH}_{4} \mathrm{OH}$ was performed by adding these products to aqueous ammonia $(1.8 \mathrm{M})$. After reacting for $15 \mathrm{hr}$ at $20^{\circ} \mathrm{C}$ the highly viscous solutions were dialyzed and the reaction products were isolated by evaporating the solvent (Ic and IIc).

The water-soluble products, obtained from the reactions of the polymerCSI adducts with $\mathrm{NaOH}$ and $\mathrm{NH}_{4} \mathrm{OH}$, appeared to be polyelectrolytes, as was shown by viscosity measurements in water.

Anticoagulant activities were determined by measuring recalcification times of bovine plasma at $37^{\circ} \mathrm{C}$ in glass tubes. Solutions of polyelectrolytes in water were added to aliquots of $1.5 \mathrm{ml}$ plasma, in amounts corresponding with concentrations of 0.04 or $0.08 \mathrm{mg} / \mathrm{ml}$ of plasma. After $5 \mathrm{~min}$ of incubation at $37^{\circ} \mathrm{C}$ recalcification was carried out with $0.3 M \mathrm{CaCl}_{2}$ to a final concentration of $1.9 \times 10^{-2} \mathrm{M}$ of added $\mathrm{CaCl}_{2}$. Heparin, $152 \mathrm{u} / \mathrm{mg}$, was purchased from Diosynth (Oss, Holland). Stock solutions of $12 \mathrm{mg} / \mathrm{ml}$ for

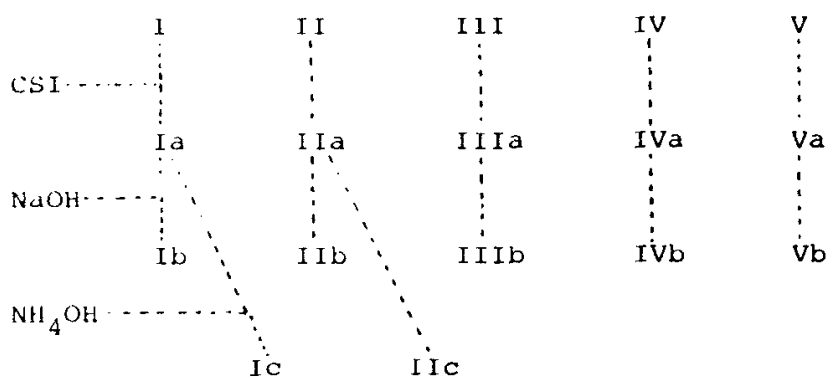

Scheme 1. Chemical modification of polymers. 
both heparin and the polyelectrolytes were used for the measurements. The $p \mathrm{H}$ of the plasma, the $0.3 \mathrm{M} \mathrm{CaCl} \mathrm{C}_{2}$ solution, and the polyelectrolyte solutions were all adjusted to 7.35 prior to use.

\section{RESULTS AND DISCUSSION}

\section{Chemical Modifications}

Previous work by Graf [7] has demonstrated that addition of CSI to the $\mathrm{C}=\mathrm{C}$ bonds in alkenes takes place (Scheme 2). This addition leads to the formation of $N$-chlorosulfonyl $\beta$-lactams (a1) and $\beta, \gamma$-unsaturated $N$ chlorosulfonyl carbonamides (a2). The ratio of the isomers (a1) and (a2) is largely determined by the substituents on the double bond, whereas the influence of the solvent has also been reported [8].

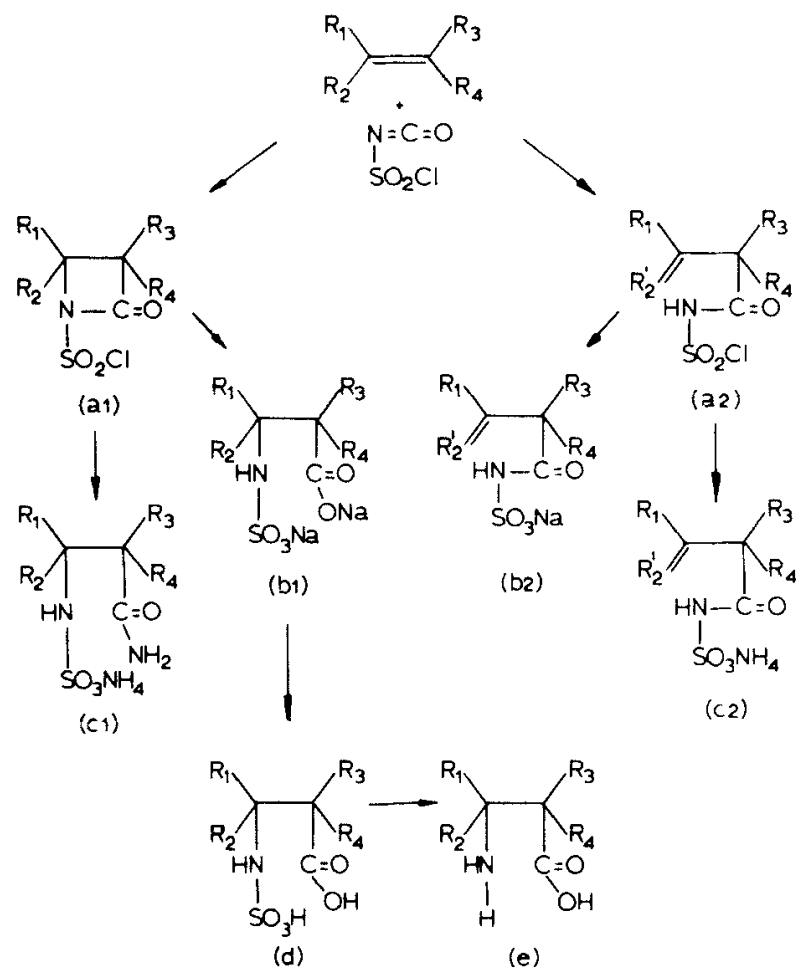

Scheme 2. Structural units in modified polymers.

The addition of CSI to $\mathrm{C}=\mathrm{C}$ bonds in polymers appeared also to be possible. With an excess of CSI, addition products were obtained as solid substances. When CSI in less than equimolar amounts was added, rubberlike products were formed. In Table I the composition of the adducts are shown, from the reaction of the polymers with an excess of CSI. 
VAN DER DOES ET AL.

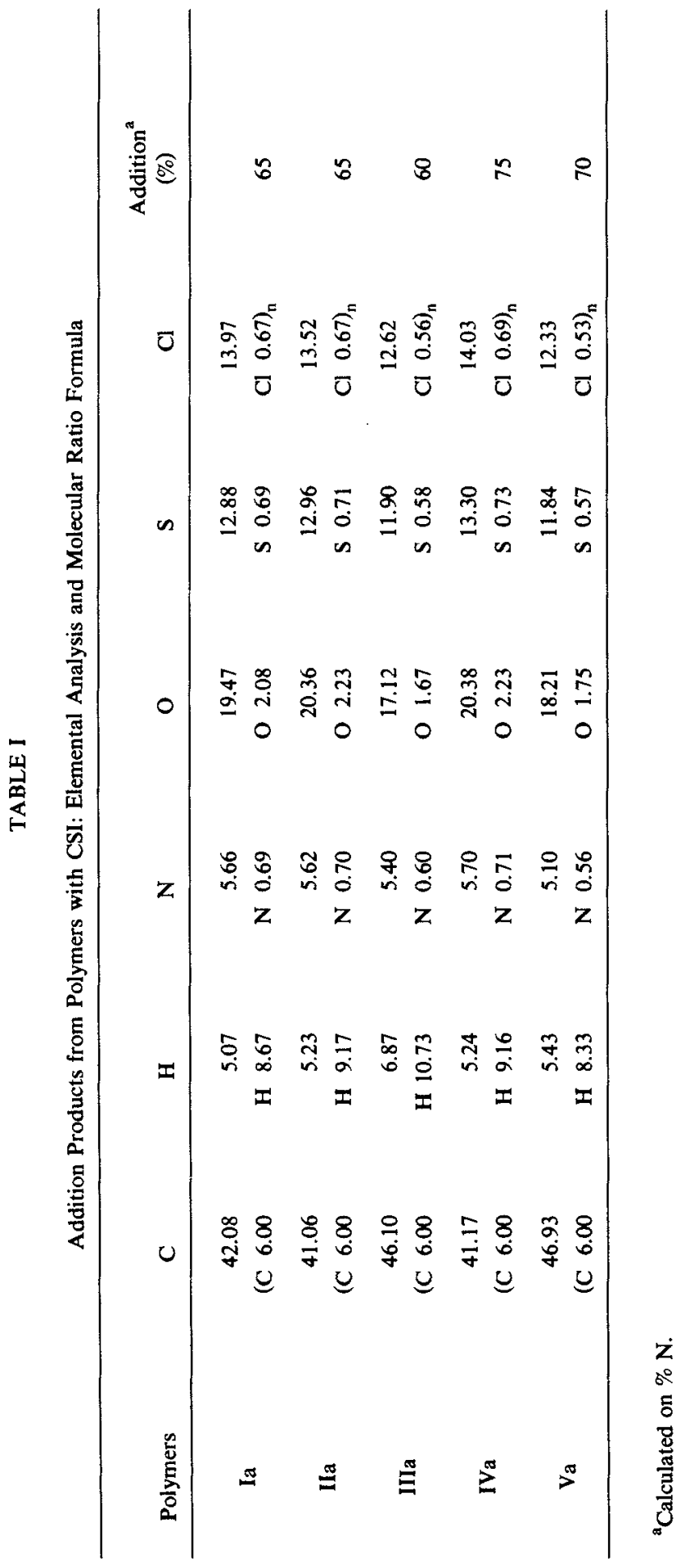


The addition of CSI to $\mathrm{C}=\mathrm{C}$ bonds should result in products with an atomic ratio of $\mathrm{N}: \mathrm{S}: \mathrm{Cl}=1: 1: 1$ and of $\mathrm{O}: \mathrm{N}=3: 1$. It can be seen from the table that the observed ratios were in fairly good agreement with the theoretical ones.

From the results of the elemental analysis it can be calculated that, in spite of the use of an excess of CSI, no more than $75 \%$ of the $\mathrm{C}=\mathrm{C}$ bonds reacted. This could be caused by the fact that during the addition reaction precipitation of the modified polymers took place, thus making further addition more difficult. It is also possible that steric hindrance prevents further addition of CSI molecules when the addition has already taken place to a certain extent.

Analysis of the ir spectra of the addition products indicated that structures (a1) and (a2) had been formed during the reactions. The spectra showed bands at 1175 and $1405 \mathrm{~cm}^{-1}$, which could be assigned to absorptions of $-\mathrm{SO}_{2}-$ groups. In addition, there were absorptions at 1810 and $1740 \mathrm{~cm}^{-1}$, which are also found in $N$-chlorosulfonyl $\beta$-lactams [9] and $\beta$, $\gamma$-unsaturated $N$-chlorosulfonyl carbonamides [10], respectively. These absorptions are attributed to the presence of $\mathrm{C}=\mathrm{O}$ groups. The absorption at $1810 \mathrm{~cm}^{-1}$ was very strong, whereas the band at $1740 \mathrm{~cm}^{-1}$ was much weaker for all of the products with the exception of IIIa. The spectra indicated that in Ia, IIa, IVa, and Va predominantly structures (a1) were present, whereas in the spectrum of IIIa absorptions characteristic for structures (a1) and (a2) had about the same intensity.

The four-membered rings in $N$-chlorosulfonyl $\beta$-lactams (a1) can be opened with $\mathrm{NaOH}$ [7] and rupture of the bonds between $\mathrm{N}$ and $\mathrm{C}=\mathrm{O}$ takes place. The $\mathrm{N}-\mathrm{S}$ bonds, however, are stable under these conditions and $N$-substituted $\beta$-amino acid salts are formed (b1). In $\beta, \gamma$-unsaturated $N$-chlorosulfonyl carbonamides (a2) the $-\mathrm{SO}_{2} \mathrm{Cl}$ groups are converted into $-\mathrm{SO}_{3} \mathrm{Na}$ groups, leading to structures (b2), whereas also bonds between $\mathrm{N}$ and $\mathrm{S}$ or $\mathrm{N}$ and $\mathrm{C}=\mathrm{O}$ can be broken [11].

The polymer-CSI adducts appeared to react similarly, as indicated by the polyelectrolyte character of the reaction products. In Table II the results of the reactions of these adducts with $\mathrm{NaOH}$ are summarized.

The $\mathbf{C}: \mathbf{N}$ and the $\mathrm{C}: \mathrm{S}$ ratios in Ib were about the same as those in Ia, whereas in the conversion of IIa into IIb the $\mathrm{C}: \mathrm{N}$ and $\mathrm{C}: \mathrm{S}$ ratio had somewhat increased. A significant increase, however, was found in the $\mathbf{C}: \mathbf{S}$ ratio when going from IIIa to IIIb, while the $\mathrm{C}: \mathrm{N}$ ratio in IIIb differed only slightly from that in IIIa.

It has been mentioned that the ir spectra of Ia and IIa indicated that structures (al) were mainly present in these polymer-CSI adducts. In the ir spectrum of IIIa, on the other hand, the bands at $1810 \mathrm{~cm}^{-1}$ and $1740 \mathrm{~cm}^{-1}$ had about the same intensity, also suggesting the presence of a reasonable fraction of structures (a2) in IIIa.

It can therefore be assumed that in the reaction of Ia and IIa with $\mathrm{NaOH}$ predominantly structures (b1) had been formed from structures (a1). 
TABLE II

Products from Polymer-CSI Adducts with $\mathrm{NaOH}$ : Elemental Analysis and Atomic Ratio

\begin{tabular}{rrrrrr}
\hline Polymers & $\mathrm{C}$ & $\mathrm{N}$ & $\mathrm{S}$ & $\mathrm{C}: \mathrm{N}$ & $\mathrm{C}: \mathrm{S}$ \\
\hline Ib & 28.25 & 3.71 & 8.59 & 8.88 & 8.77 \\
Ia & & & & 8.70 & 8.70 \\
IIb & 30.29 & 3.91 & 8.73 & 9.04 & 9.25 \\
IIa & & & & 8.57 & 8.45 \\
IIIb & 34.08 & 3.78 & 5.95 & 10.52 & 15.27 \\
IIIa & & & & 10.00 & 10.34 \\
IVb & 52.18 & 5.77 & 5.41 & 10.57 & 25.72 \\
IVa & & & & 8.45 & 8.22 \\
Vb & 56.34 & 5.23 & 4.73 & 12.55 & 31.80 \\
Va & & & & 10.71 & 10.53 \\
& & & & & \\
\hline
\end{tabular}

Probably, modification of IIIa had resulted in the formation of structures (b1) as well as cleavage of the $\mathrm{N}-\mathrm{S}$ bonds in structures (a2), thus leading to the great change in the $\mathrm{C}: \mathrm{S}$ ratio.

Based upon this hypothesis it can be calculated that the isomeric structures (a1) and (a2) in IIIa had been formed in a ratio of about $2: 1$. Obviously, the N-S bonds in structures (a1) were quite stable in the ringopening reaction, because only a slight to moderate increase in the $\mathrm{C}: \mathrm{S}$ ratio in Ia and IIa was observed.

After reaction of IVa and $\mathrm{Va}$ with $\mathrm{NaOH}$ no precipitate was formed upon addition of the reaction mixtures to ethanol. It was possible, however, to isolate $\mathrm{IVb}$ and $\mathrm{Vb}$ by precipitation with concentrated $\mathrm{HCl}$.

From Table II it can be seen that the $\mathrm{C}: \mathrm{N}$ and $\mathrm{C}: \mathrm{S}$ ratios in $\mathrm{IVb}$ and $\mathrm{Vb}$ differed considerably from those in IVa and $\mathrm{Va}$, respectively. In the ir spectra of IVa and Va the ratios of the intensities of the absorptions at 1810 and $1740 \mathrm{~cm}^{-1}$ were the same as in Ia. Therefore it was expected that mainly structures (b1) had been formed from IVa and Va.

The ir spectra of IVb and $\mathrm{Vb}$ were identical, but quite different from the spectrum of $\mathrm{Ib}$ (Fig. 1). The absorptions at $1560 \mathrm{~cm}^{-1}$ as present in $\mathrm{Ib}$, characteristic for carboxylate groups [structures (b1)], were absent in the spectra of $\mathrm{IVb}$ and $\mathrm{Vb}$. A strong peak was visible at $1705 \mathrm{~cm}^{-1}$, which could be attributed to absorptions of carboxylic acid groups. It was also found that in $\mathrm{IVb}$ and in $\mathrm{Vb}$ no sodium was present and it was therefore concluded that upon acidification of the reaction mixtures, conversion of structures (b1) into structures (d) had taken place.

The increase in the $\mathrm{C}: \mathrm{N}$ ratio in IVb and in $\mathrm{Vb}$ indicated that part of the $\mathrm{C}-\mathrm{N}$ bonds in structures (d) had been broken. This cleavage occurred for $15-20 \%$ in $\mathrm{IVb}$ as well as in $\mathrm{Vb}$. It can further be calculated that an 


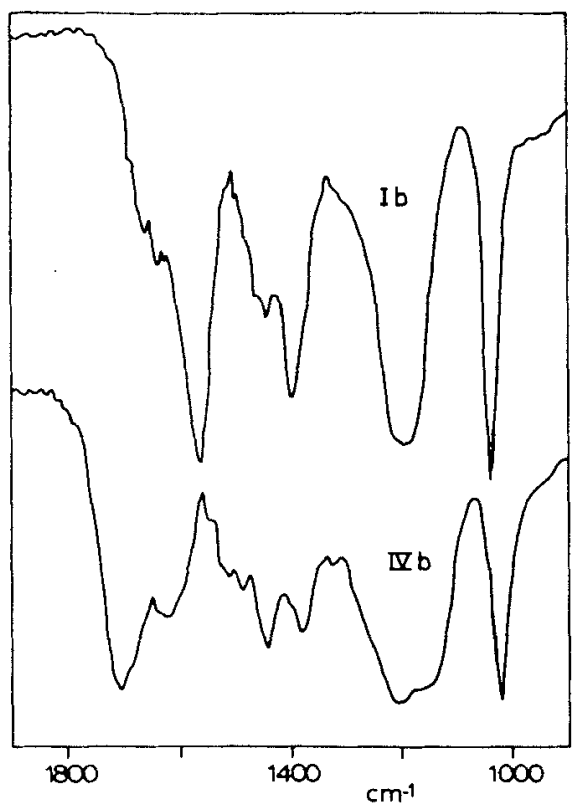

FIG. 1. Infrared spectra of products from the reaction of polymer-CSI adducts with $\mathrm{NaOH}$.

increase of $65-70 \%$ of the $\mathrm{C}: \mathrm{S}$ ratio had occurred in the conversion of both IVa and Va.

Evidently, in this acidic medium part of the $\mathrm{N}-\mathrm{S}$ bonds in structures (d) had also been broken, resulting in the formation of structures (e).

Rupture of N-S bonds also occurred upon acidification of an aqueous solution of Ib. It appeared that after stirring $\mathrm{Ib}$ for $200 \mathrm{hr}$ in $0.1 \mathrm{M} \mathrm{HCl}$ the original $\mathbf{N}: \mathrm{S}$ ratio of $1: 1$ had increased to $2: 1$. The resulting product $\mathrm{Ib}(\mathrm{s})$ contained no sodium and in the ir spectrum the peak at $1560 \mathrm{~cm}^{-1}$ had been replaced by an absorption at $1705 \mathrm{~cm}^{-1}$. There was practically no change in the $\mathrm{C}: \mathrm{N}$ ratio, probably owing to the mildly acidic conditions. Obviously conversion of structures (b1) into (d) had taken place in this reaction, whereas subsequently part of the $\mathrm{N}-\mathrm{S}$ bonds had been broken.

The reaction of $N$-chlorosulfonyl $\beta$-lactam groups in polymers with aqueous ammonia has been reported recently [3]. A ring-opening reaction was supposed to occur in a similar way as the reaction of $N$-chlorosulfonyl $\beta$-lactams with alifatic and aromatic amines [7]. Table III gives more details about the reaction products resulting from the conversion of Ia and IIa into Ic and IIc, respectively.

If only conversion of structures (a1) into structures (c1) had taken place in the reaction with ammonia, no change of the $\mathrm{C}: \mathrm{S}$ ratio should result, whereas the $\mathrm{C}: \mathrm{N}$ ratio should diminish with a factor 3 . It can be seen from the table that the $\mathrm{C}: \mathrm{S}$ ratio in Ic was nearly the same as in Ia, while 
TABLE III

Products from Polymer-CSI Adducts with $\mathrm{NH}_{4} \mathrm{OH}$ :

Elemental Analysis and Atomic Ratio

\begin{tabular}{lcccccc}
\hline Polymers & $\mathrm{C}$ & $\mathrm{N}$ & $\mathrm{S}$ & & $\mathrm{C}: \mathrm{N}$ & $\mathrm{C}: \mathrm{S}$ \\
\hline & & & & obs. & calc. & \\
Ic & 40.12 & 15.13 & 11.84 & 3.09 & 2.90 & 9.04 \\
Ia & & & & 8.70 & & 8.70 \\
Ilc & 41.35 & 14.17 & 11.00 & 3.40 & 2.86 & 10.02 \\
IIa & & & & & & 8.57 \\
\hline
\end{tabular}

in IIc evidently part of the $\mathrm{N}-\mathrm{S}$ bonds has been broken. The observed $\mathrm{C}$ : $\mathrm{N}$ ratios, on the other hand, were not very different from the calculated ones. These results indicate that in these conversions the formation of structures (c2) was negligible, in agreement with the assumption that in Ia and in IIa structures (a2) were nearly absent.

Both Ic and IIc are soluble in water, and viscosity measurements showed their polyelectrolyte character. In the ir spectra, the $1810-\mathrm{cm}^{-1}$ and $1740-\mathrm{cm}^{-1}$ bands had disappeared and a new absorption at $1655 \mathrm{~cm}^{-1}$ was present, which could be assigned to carbonamide absorption. The absence of carboxylate groups was further indicated by analysis of acidified solutions of Ic and IIC in water. The ir spectra of the isolated products still showed absorptions at $1655 \mathrm{~cm}^{-1}$, whereas a band at $1705 \mathrm{~cm}^{-1}$ should have been visible if in Ic and in IIc carboxylate groups were present.

Summarizing, it can be assumed that in the reaction of Ia and IIa with aqueous ammonia conversion had taken place of structures (a1) into (c1) and, possibly, of structures (a2) into (c2).

\section{Anticoagulant Activity}

The anticoagulant activity of $\mathrm{Ib}$ has been reported recently $[4,5]$. It was shown that $\mathrm{Ib}$ is an antithrombin and that its action, like heparin, depends on a cofactor (presumably antithrombin III), present in the blood plasma.

In Figure 2 the structure of heparin is shown [12], and it can be seen that a structural resemblance exists between $\mathrm{Ib}$ and heparin because of the presence of the sulfamate (- $\mathrm{NHSO}_{3}{ }^{-}$) and carboxylate groups. From several studies it has been concluded that these groups are essential for the activity of heparin [12]. Therefore, the presence of these groups in Ib was supposed to be responsible for the observed anticoagulant activity. 


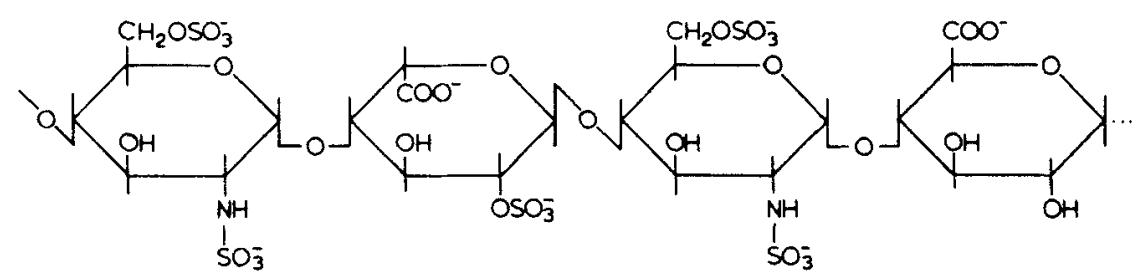

FIG. 2. Structure of heparin (12).

In order to test the validity of this hypothesis, the behavior of other polyelectrolytes was studied, the synthesis of which has been described in the previous section.

In Table IV the anticoagulant activity of the various polyelectrolytes is shown. At concentrations of $0.08 \mathrm{mg} / \mathrm{ml}$ plasma the difference in activity of these compounds is quite apparent.

In an attempt to increase the activity of $\mathrm{Ib}$, this product was dialyzed against water. A product was obtained in a yield of $70 \%$ [Ib(d)], which was considerably more active than $\mathrm{Ib}$. By fractionation of $\mathrm{Ib}$ with ethanolwater mixtures, a method described for the fractionation of heparin [13], a product could be isolated as active as $\mathrm{Ib}(\mathrm{d})$, but in a much lower yield.

It has been mentioned earlier that the $\mathrm{N}-\mathrm{S}$ bonds in $\mathrm{Ib}$ could be broken under acidic conditions. As a consequence the number of sulfamate groups in Ib would decrease and possibly the anticoagulant activity would be influenced.

Therefore the activity was determined of a product $\mathrm{Ib}(\mathrm{s})$, obtained after cleavage of $50 \%$ of the original $\mathrm{N}-\mathrm{S}$ bonds in Ib. It was found that the increase of the $N: S$ ratio had resulted in a complete disappearance of the anticoagulant activity.

The sulfamate groups in Ib appeared therefore to be essential for the anticoagulant activity, as has also been found for heparin. In addition it has to be mentioned that although $50 \%$ of the sulfamate groups is still present in $\mathrm{Ib}(\mathrm{s})$, this obviously is not sufficient to show anticoagulant activity.

In this respect it is interesting to note that only after $30 \%$ of the N-S bonds in heparin had been broken did a major decrease in anticoagulant activity occur [14]. It may be possible, too, that in Ib a lower degree of $\mathrm{N}-\mathrm{S}$ bond cleavage than $50 \%$ is tolerable before a loss of activity will be found.

The number of sulfamate and carboxylate groups in IIb was about the same as in Ib, but the activity was about half of that of Ib. Dialysis of IIb also led to an increase of the activity [IIb(d)], but the effect was not as significant as was found for Ib. Evidently, not only the presence of a sufficient number of sulfamate (and possibly carboxylate) groups is necessary to show anticoagulant activity, but the spatial position of these groups in the molecules seems to be important as well. 


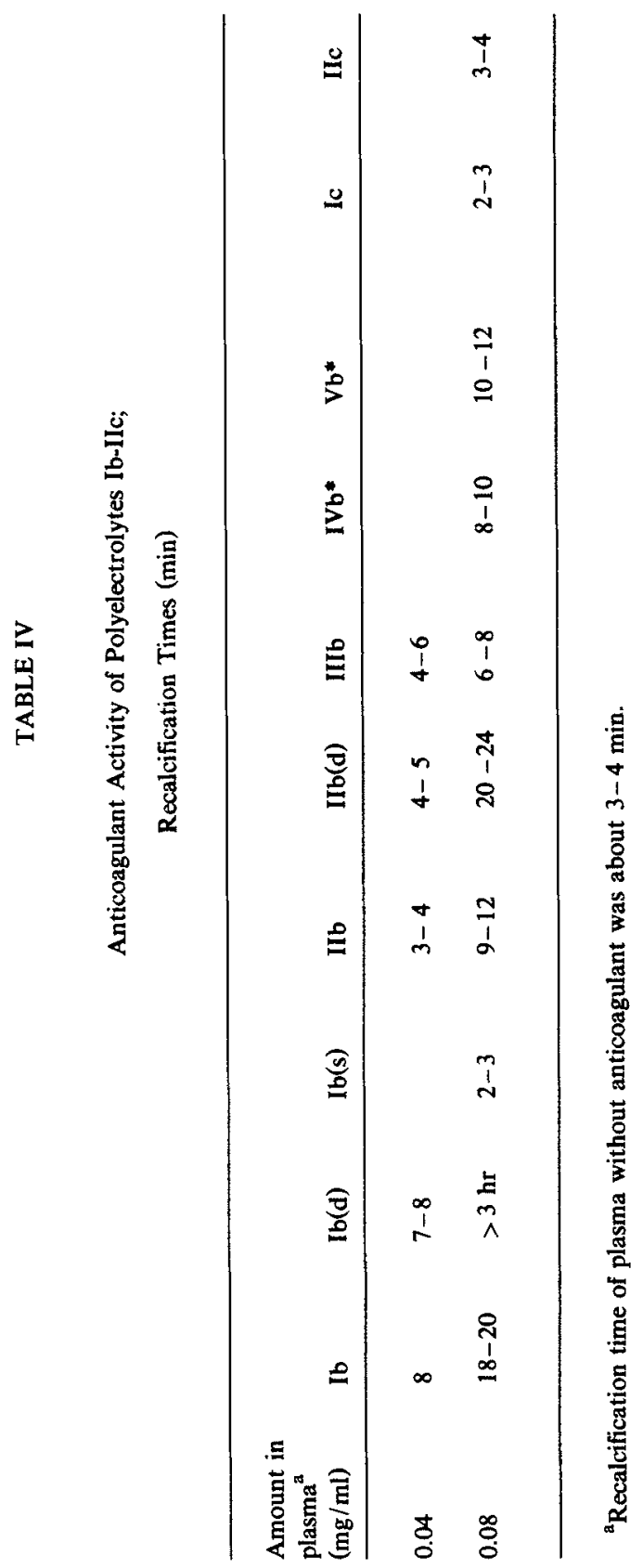


TABLE V

Anticoagulant Activity of Ib(d) Compared with Heparin

\begin{tabular}{cccc}
\hline $\begin{array}{c}\text { Coagulation } \\
\text { after min }\end{array}$ & $\begin{array}{c}\text { Heparin : plasma } \\
10^{-4} \mathrm{mg} / \mathrm{ml}\end{array}$ & $\begin{array}{c}\mathrm{Ib}(\mathrm{d}): \text { plasma } \\
10^{-3} \mathrm{mg} / \mathrm{ml}\end{array}$ & $\begin{array}{c}\text { Activity } \\
\text { ratio }\end{array}$ \\
\hline 3.30 & 15.4 & 21.2 & 0.07 \\
5 & 18.8 & 27.6 & 0.07 \\
7 & 21.7 & 33.2 & 0.07 \\
10 & 24.0 & 38.4 & 0.06 \\
\hline
\end{tabular}

The activity of IIIb was about $1 / 3$ compared with $\mathrm{Ib}$. This difference may be due to the fact that in IIIa structures (a1) and (a2) were present. Hydrolysis of structures (a2) obviously resulted in N-S splitting, and possibly the remaining sulfamate groups in structures (b1) were not sufficient to show anticoagulant activity. It is also possible that the different spatial position of the sulfamate (and carboxylate) groups in IIIb compared with Ib is responsible for the different behavior of the two polyelectrolytes.

From the elemental analysis of $\mathrm{IVb}$ and $\mathrm{Vb}$ it could be calculated that an increase of about $65-70 \%$ of the $\mathrm{C}: \mathrm{S}$ ratios had taken place. These products had no anticoagulant activity, as was expected. The data for IVb* and $\mathrm{Vb}^{*}$ in Table IV were obtained from experiments after heating of IVa and $\mathrm{Va}$ with $\mathrm{NaOH}$ for $10 \mathrm{hr}$, and subsequent precipitation of the products with ethanol.

It can be seen that $\mathrm{IVb}^{*}$ and $\mathrm{Vb}^{*}$ show activity, but they are not as active as $\mathrm{Ib}$. This result was unexpected, because the degree of addition of CSI to IV and V was higher than to I. In addition the spatial position of the sulfamate and carboxylate groups in IVb* and $\mathrm{Vb}^{*}$ is the same as in Ib.

It has been reported that the anticoagulant activity of heparin decreases upon heating in an alkaline medium as the result of depolymerization [15]. Possibly the activities of $\mathrm{IVb}^{*}$ and $\mathrm{Vb}^{*}$ are low, owing to the prolonged heating of these products.

Because in heparin both the sulfamate and carboxylate groups are essential for the anticoagulant activity, it was of interest to compare the activities of Ic and IIc with those of Ib and IIb, respectively. The difference between these polyelectrolytes is that the carboxylate groups in Ib and IIb have been substituted by carbonamide groups (Ic and IIc, respectively).

From Table IV it can be seen that Ic and IIc show no activity. Apparently the activity has disappeared as a result of the substitution of the $-\mathrm{ONa}$ groups by $-\mathrm{NH}_{2}$ groups, because in Ic and in IIc about the same 
number of sulfamate groups are present as in Ib and IIb, respectively (cf. the $\mathrm{C}: \mathrm{S}$ ratios in Table II and III). Thus, the anticoagulant activity seems also to be dependent on the presence of carboxylate groups in these polyelectrolytes.

The results in Table IV show that $\mathrm{Ib}(\mathrm{d})$ is the most active anticoagulant. By measuring recalcification times of bovine plasma in the presence of a polyelectrolyte, it was found that $\mathrm{Ib}(\mathrm{d})$ had an activity of 6-7\% compared with heparin (Table V). However, determination of the activated clotting time of blood from rabbits which were injected with $\mathrm{Ib}(\mathrm{d})$ showed that the in vivo activity is $50 \%$ in comparison with heparin.

From these investigations it can be concluded that the combination of sulfamate and carboxylate groups which are placed in distinct spatial positions seems to be essential for the anticoagulant activity of the polyelectrolytes, and in this respect the resemblance with heparin is striking.

The authors are indebted to Ms. W. Kemper, Mr. J. J. Bresser, Mr. F. A. Kuypers, Mr. G. J. Met, and Mr. L. C. Sederel for experimental assistance. Thanks are also due to Mr. J. C. F. de Jong, Department of Experimental Surgery, State University of Groningen, The Netherlands, for determining the in vivo anticoagulant activity.

\section{REFERENCES}

[1] R. Pautrat and J. Marteau, Rev. Gen. Caoutch. Plost., 48, 1227 (1971).

[2] L. van der Does, J. Hofman, and T. E. C. van Utteren, J. Polym. Sci. Polym. Lett. Ed., 11, 169 (1973).

[3] P. M. van der Velden, M. H. V. Mulder, L. van der Does, and C. A. Smolders, J. Polym. Sci. Polym. Lett. Ed., 14, 5 (1976).

[4] T. Beugeling, L, van der Does, A. Bantjes, and W. L. Sederel, J. Biomed. Mater. Res, 8 , 375 (1974).

[5] T. Beugeling, L. van der Does, B. V. Rejda, and A. Bantjes, in Biocompatibility of Implant Materials, D. Williams, Ed., Sector, London, 1976, p. 187.

[6] T. Beugeling, J. Feijen, A. H. J. Tijhuis, P. E. Froehling, M. A. de Jongh, M. Looze-van Iperen, and A. Bantjes, Proc. Eur. Soc. Artif. Organs, 3, 76 (1976).

[7] R. Graf, Justus Liebigs Ann. Chem., 661, 111 (1963); Angew. Chem., 80, 179 (1968).

[8] C. P. Pinazzi, P. Noireaux, and D. Reyx, Makromol. Chem., 175, 2849 (1974).

[9] P. Goebel and K. Clauss, Justus Liebigs Ann. Chem., 722, 122 (1969).

[10] Hi. Destian, H. Biener, K. Clauss, and H. Heyn, Justus Liebigs Ann. Chem. 718, 94 (1968).

[11] A. Dorlars, in Methoden der Organischen Chemie, E. Müller, Ed., Thieme, Stuttgart, Vol. $\mathrm{XI} / 2,1958$, p. 641.

[12] I. Danishefsky, Adv. Exp. Med. Biol., 52, 105 (1975).

[13] S. E. Lasker and S. S. Stivala, Arch, Biochem. Biophys, 115,360 (1966).

[14] J. A. Cifonelli, Adv. Exp. Med. Biol, 52, 95 (1975).

[15] J. E. Jorpes, H. Boström, and V. Mutt, J. Biol. Chem., 183, 607 (1950). 